\title{
A Deep Convolutional Neural Network for Segmenting and Classifying Epithelial and Stromal Regions in Histopathological Images
}

\author{
Jun $\mathrm{Xu}^{\mathrm{a}, *}$, Xiaofei Luo ${ }^{\mathrm{a}}$, Guanhao Wang ${ }^{\mathrm{a}}$, Hannah Gilmore ${ }^{\mathrm{b}}$, Anant \\ Madabhushic \\ ${ }^{a}$ Jiangsu Key Laboratory of Big Data Analysis Technique, Nanjing University of \\ Information Science and Technology, Nanjing 210044, China. \\ ${ }^{b}$ Institue for Pathology, University Hospitals Case Medical Center, Case Western \\ Reserve University, $\mathrm{OH}$ 44106-7207, USA \\ ${ }^{c}$ Dept. of Biomedical Engineering, Case Western Reserve University, OH44106, USA.
}

\begin{abstract}
Epithelial (EP) and stromal (ST) are two types of tissues in histological images. Automated segmentation or classification of EP and ST tissues is important when developing computerized system for analyzing the tumor microenvironment. In this paper, a Deep Convolutional Neural Network$\mathrm{s}$ (DCNN) based feature learning is presented to automatically segment or classify EP and ST regions from digitized tumor tissue microarrays (TMAs). Current approaches are based on handcraft feature representation, such as color, texture, and Local Binary Patterns (LBP) in classifying two regions. Compared to handcrafted feature based approaches, which involve task dependent representation, DCNN is an end-to-end feature extractor that may be directly learned from the raw pixel intensity value of EP and ST tissues in a data driven fashion. These high-level features contribute to the construction of a supervised classifier for discriminating the two types of tissues. In this work we compare DCNN based models with three handcraft feature extraction based approaches on two different datasets which consist of 157 Hematoxylin and Eosin (H\&E) stained images of breast cancer and 1376 immunohistological (IHC) stained images of colorectal cancer, respectively. The DCNN based feature learning approach was shown to have a F1 classification
\end{abstract}

\footnotetext{
${ }^{*}$ Corresponding author

Email address: xujung@gmail.com (Jun Xu)
} 
score of $85 \%, 89 \%$, and $100 \%$, accuracy (ACC) of $84 \%, 88 \%$, and $100 \%$, and Matthews Correlation Coefficient (MCC) of $86 \%, 77 \%$, and $100 \%$ on two $\mathrm{H} \& \mathrm{E}$ stained (NKI and VGH) and IHC stained data, respectively. Our DNN based approach was shown to outperform three handcraft feature extraction based approaches in term of the classification of EP and ST regions.

Keywords: Deep convolutional neural networks; feature representation; the classification of epithelial and stromal regions; breast histopathology; colorectal cancer.

\section{Introduction}

Stromal (ST) tissue includes the fatty and fibrous connective tissues surrounding the ducts and lobules, blood vessels, and lymphatic vessels, which are supportive framework of an organ. Epithelial (EP) tissue is the cellular tissue lining and found in the ductal and lobular system of the breast milk ducts. About $80 \%$ breast tumors originate in the breast EP cells. Although ST tissue is typically considered as not being part of malignant tissue, the changes in the stroma tend to drive tumor invasion and metastasis [11]. Therefore, tumor-stroma ratio in histological tissues is being recognized as an important prognostic value [12], since cancer growth and progression is dependent on the microenvironment of EP and ST tissues. Yuan et al. in [31] found that the spatial arrangement of stromal cell in tumors is a prognostic factor in breast cancer. Consequently a critical initial step in developing automated computerized algorithms for risk assessment and prognosis determination is to be able to distinguish stromal from epithelial tissue compartments on digital pathology images. This is however extremely challenging due to the high data density, the complexity of the tissue structures, and the inconsistencies in tissue preparation. Therefore, it is crucial to develop intelligent algorithms for the segmentation of different tissue structures in an accurate, fast, practical and robust manner [25, 32, 33, 34].

\section{Previous Works}

There has been substantial interest recently in developing approaches for automated classification of stromal and epithelial regions within $\mathrm{H} \& \mathrm{E}$ tissue images. In [19], local binary pattern (LBP) and contrast measure based texture features were used for discriminating epithelium and stroma from 
immunohistochemistry (IHC) stained tumor tissue microarrays (TMAs) of colorectal cancer. Five perception-based features (coarseness, contrast, directionality, line-likeiness and roughness), features related to human perception, were presented in [6] to differentiate EP and ST patches [19]. In [14], color based texture features extracted from square image blocks for automated segmentation of stromal tissue from IHC images of breast cancer. A binary graph cuts approach where the graph weights were determined based on the colour histogram of two regions, was used for segmenting EP and ST regions from odontogenic cysts images in [13]. In [17], a cell graph feature describing the topological distribution of the tissue cell nuclei was used for discriminating tumor and stromal areas on immunofluorescence histological images. In [3], IHC stained TMA cores were automatically stratified as tumor or non-tumor cores based on a visual word dictionary learning approach. As LBP based approaches can only deal with gray scale images, in [19], prior to feature extraction, each color image is converted into gray scale images by computing a weighted sum of $\mathrm{R}, \mathrm{G}$, and $\mathrm{B}$ components. However since the conversion assumes that each pixel in the gray scale image is a linear combination of three color components, an assumption that is not always true, LBP features could be derived off sub-optimal image representations.

The fixed-size window or pixel-grid is one of the traditional ways to select patches from bigger images prior to feature extraction. Recently, superpixel based approaches [23] are being employed to group pixels into meaningful atomic regions based on similarity. Two popular superpixel algorithms are Normalized Cut (Ncut)-based [23, 24] and Simple Linear Iterative Clustering (SLIC)-based [1]. Ncut-based superpixel algorithm essentially employs graph theory to explore the pixel-wise similarity among the pixels being interrogated and their neighbourhood pixels. The SLIC-based superpixel algorithm is based on clustering and employs the similarity of each pixel's color and Euclidean distance. Ncut-based superpixel algorithm is more accurate but is more computationally intensive. Compared to Ncut-based algorithm, the SLIC-based approach is simple and faster, but is less accurate. Compared to traditional pixel-grids, the atomic regions generated via a superpixel algorithm represent a natural partitioning of visual scenes. As different tissue structures are mutually present in histologic images, superpixel based approaches are often employed as a pre-processing step to mitigate the issue of possible over-segmentation of the tissue images into atomic regions. The atomic regions are then subsequently segmented into epithelial and stromal regions. In [4], a superpixel based algorithm was used to over-segment breast 
tissue hematoxylin and eosin (H \& E) images into small compartments. Subsequently the cell nuclei and cytoplasm within each smaller subcompartment were further classified into epithelial and stromal regions by a Support Vector Machine (SVM) classifier. Similarly, a superpixel based SVM was employed to separate EP from ST areas in tissue regions of oropharyngeal squamous cell carcinoma in [2].

All the previously proposed methods were based off handcrafted features such as color and texture which aim to simulate the visual perception of human pathologist in interpreting the tissue samples [30]. Recently, however, there has been interested "deep learning" (DL) strategies for classification and analysis of big data. Histopathology, given the data complexity and density, is ideally aligned with deep learning approaches that attempt to use deep architectures to learn complex features from data. DL approaches unlike handcrafted feature approaches represent end-to-end feature learning approach which attempt to learn high-level structural features from a large amount of training data to best discriminate between the classes of interest. The DL approach can thus serve as a good feature extractor for better data representation [18]. In [9], a deep max-pooling convolutional neural network was presented for detecting mitosis in breast histological images. The approach comprised a deep neural network involving a convolutional and a max-pooling layer which were employed to learn the representation of high-level features. Then, a supervised softmax classifier was trained to classify each pixel within a square window as containing a mitotic nucleus or not. In [10], a convolutional neural networks (CNN) and autoencoder were combined for histopathological image representation based learning. Then a softmax classification approach was employed for distinguishing cancerous and non-cancerous tissue. The approach in [10] used a one-layer autoencoder for high-level feature representation. In [28, 29], we presented a Stacked Sparse Autoencoder (SSAE) framework for automated nuclear detection from high resolution breast histopathological images. Handcrafted features were combined with CNN features in [26] for mitosis detection in breast cancer pathology. DCNN is a hierarchical neural network which mimics the network structure of neural systems. It is a multi-layer network of interconnected simple "neurons" by connecting links characterized by a weight.

Building on these approaches, in this work, we present a patch based DCNN approach for distinguishing epithelial and stromal compartments within H\&E images of breast cancers [8]. Each histologic image is first represented by thousands of cropped sub-images. Two different approaches involving the 
use of superpixel (SP) and a fixed-size square window is used to generate subimages from $\mathrm{H} \& \mathrm{E}$ and IHC stained images, respectively. Different from color or intensity based features, such as LBP [19] and texture [6], our approach employs architectural features of atomic regions in the tumor and stroma for tissue classification. The DCNN based feature learning is applied to two classifications of EP and ST patches on (1) IHC stained histologic images of colorectal cancer and (2) on H\&E stained images of breast cancer. For simplicity, throughout this paper, we use two different terms "Classification" and "Segmentation" to represent the two different applications, respectively. The classification of EP and ST patches of IHC stained images is an easier task which aims to assign a single label to the respective patch. Segmentation of EP and ST regions is more difficult since it aims to detect the regions of interest (ROIs) and then assign a label to each corresponding ROI. For the classification task, we employed a fixed-size SW to extract candidate subimages defined via a sliding window scheme. These are then fed to the DCNN for training the network. The flowchart for the classification framework with DCNN is shown in Figures $2(\mathrm{~g})-(\mathrm{k})$. As the separation of the epithelial and stromal regions from $\mathrm{H} \& \mathrm{E}$ images is a more difficult task, we firstly employ a superpixel based scheme to over-segment the image into atomic regions. Then the atomic regions are resized into fixed-size square images, prior to feeding them to a DCNN for feature learning.

The rest of this paper is organized as follows. A detailed description of DCNN is presented in Section 3. The experimental setup and comparative strategies are presented in Section 4. The experiment results and a discussion of the results are reported in Section 5. Concluding remarks are presented in Section 6.

\section{Methods}

\subsection{The Deep Convolutional Neural Networks (DCNN)}

The DCNN approach employed in this paper comprises two alternating convolutional layers (or $\mathcal{C}$ layers, see Figure 1(b)), max-pooling (or $\mathcal{P}$ layers, see Figure 1(c)), two full connection layers, and a final classification layer. The $\mathcal{C}$ and $\mathcal{P}$ layers produce a convolution and a max-pooling feature map via successive convolution and max-pooling operations, respectively. These feature maps then enable the extraction and combination of a set of appropriate image features from the training exemplars. 


\begin{tabular}{|c|c|c|c|}
\hline Symbol & Description & Symbol & Description \\
\hline DCNN & Deep Convolutional Neural Networks & $\mathrm{SMC}$ & Softmax Classifier \\
\hline $\mathrm{EP}$ & Epithelial region & $\mathrm{ST}$ & Stromal region \\
\hline LBP & Local Binary Patterns & SVM & Support Vector Machine \\
\hline F1 & F1 score & $\mathrm{ACC}$ & Accuracy \\
\hline $\mathrm{TP}$ & True Positive & $\mathrm{FP}$ & False Positive \\
\hline $\mathrm{FN}$ & False Negative & $\mathrm{TN}$ & True Negative \\
\hline MCC & Matthews Correlation Coefficient & $\mathrm{ROC}$ & Receiver Operating Characteristic \\
\hline TMAs & Tumor Tissue Microarrays & IHC & Immunohistochemistry \\
\hline $\mathrm{H} \& \mathrm{E}$ & Hematoxylin and Eosin & DL & Deep Learning \\
\hline $\mathrm{SP}$ & Superpixel & SW & Sliding Window \\
\hline $\mathcal{C}$ layer & Convolutional layer & $\mathcal{P}$ layer & max-pooling layer \\
\hline $\mathcal{F C}$ layer & Full Connection layer & ROI & Region of Interest \\
\hline$D_{1}$ & Dataset 1 & $D_{2}$ & Dataset 2 \\
\hline NKI & Netherlands Cancer Institute Dataset & VGH & Vancouver General Hospital Dataset \\
\hline SLIC & Simple Linear Iterative Clustering algorithm & Ncut & Normalized Cuts algorithm \\
\hline $\mathrm{DCNN}+\mathrm{SMC}$ & DCNN plus SMC & $\mathrm{DCNN}+\mathrm{SVM}$ & DCNN plus SVM \\
\hline
\end{tabular}

Table 1: Enumeration of the symbols used in the paper.

\subsection{The convolutional layer (C layer)}

Let $W^{l}=\left(w_{1}^{l}, w_{2}^{l}, \ldots, w_{d_{h}^{l}}^{l}\right)$ be a filter bank. Each $w_{k}^{l}, k \in\left\{1,2, \ldots, d_{h}^{l}\right\}$, is an $m^{l} \times m^{l}$ linear filter embedded in the $l$-th layer. $d_{h}^{l}$ is the number of different filters or kernels in the filter bank $W^{l}$. An input $\omega^{l-1} \times \omega^{l-1}$ patch $I_{p}^{l-1}$ is convolved with a $m^{l} \times m^{l}$ local receptive region in the image $I_{p}^{l-1}$ encompassed by the filter $w_{k}^{l}$. The filter $w_{k}^{l}$ moves over the input patch $I_{p}^{l}$ and allows for a local convolution operation. For each image patch extracted from the image, the convolutional operations with $d_{h}^{l}$ filters result in $d_{h}^{l}$ feature maps. The result of the linear convolutional operation on $I_{p}^{l-1}$ by $w_{k}^{l}$ can be re-written as $g_{k}^{l}=I_{p}^{l-1} * w_{k}^{l}$. Here the pixel value $g_{k}(p)$ of each location of the resultant image $g_{k}^{l}$ is the sum of products of the filter coefficients and the image pixels of the local receptive region encompassed by the filter. During the convolutional operations, each filter $w_{k}$ convolves across $\left(\omega^{l-1}-m^{l}+1\right) \times$ $\left(\omega^{l-1}-m^{l}+1\right)$ pixels in $I_{p}^{l-1}$. The size of the resultant images $g_{k}^{l}$ is therefore $\left(\omega^{l-1}-m^{l}+1\right) \times\left(\omega^{l-1}-m^{l}+1\right)$ following the convolutional operation. $g_{k}^{l}$ is the pre-activation or input to the nonlinear activation function of a neuron. The output of the active function is $x^{l}=f\left(g_{k}^{l}\right)=\frac{1}{1+\exp \left(-g_{k}^{l}\right)}=\frac{1}{1+\exp \left(-I_{p}^{l-1} * w_{k}^{l}\right)}$. The procedure of convolutional operation is illustrated in Figure 1 (b).

\subsection{The max-pooling layer ( $\mathcal{P}$ layers)}

The max-pooling layers or $\mathcal{P}$ layers aim to achieve spatial invariance by reducing the resolution of the feature maps obtained in layer $\mathcal{C}$. It applies 


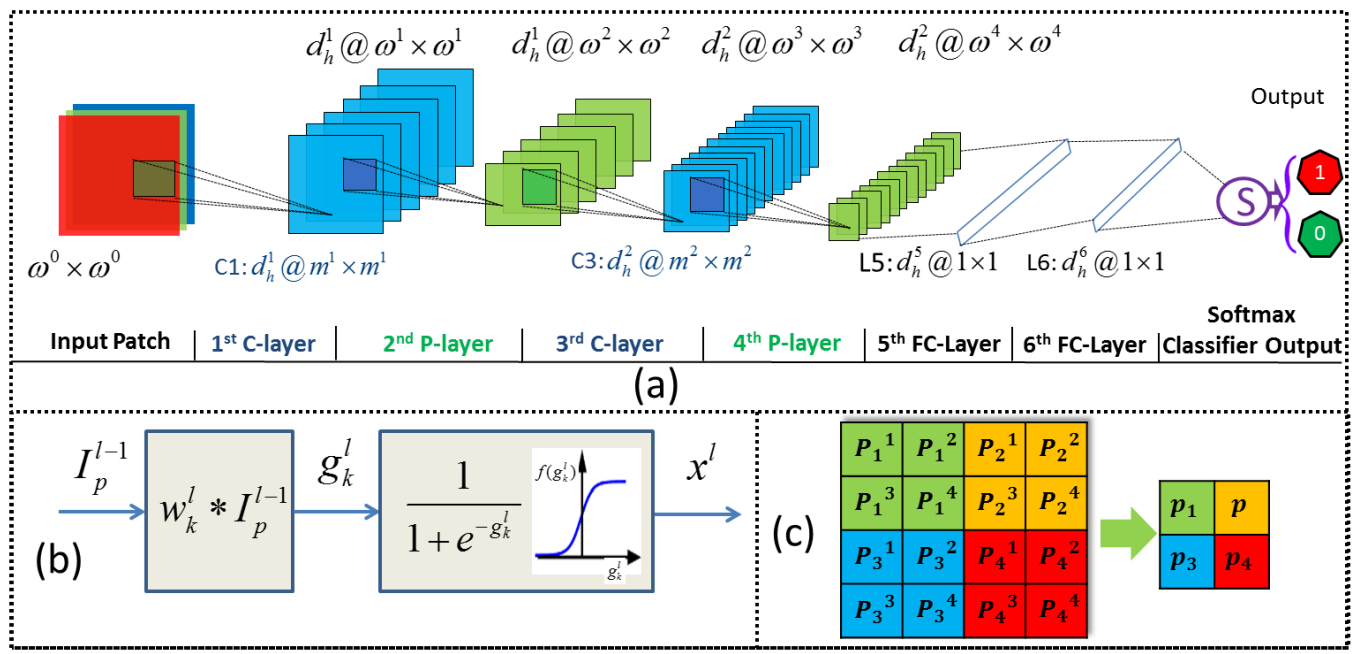

Figure 1: The architecture of the new DCNN employed in this work. The approach comprises of (a) two alternating convolutional layers (or $\mathcal{C}$-layers) with the convolutional operation (b) and max-pooling layers (or $\mathcal{P}$-layers) with the max-pooling operation, and (c) two full connection layers (or $\mathcal{F C}$ layers), and an output layer.

local pooling of feature maps using a max operation in the neighbourhood of the results of the $\mathcal{C}$ layer. The max-pooling operation in $\mathcal{P}$ layers is described in Figure 1 (c). Subsequent to the max-pooling operation, the size of the resultant images becomes $\frac{\left(\omega^{l-1}-m^{l}+1\right)}{s} \times \frac{\left(\omega^{l-1}-m^{l}+1\right)}{s}$, where $s$ refers to the scale at which the operation was applied.

\subsection{Output layer: Softmax Classifier (SMC)}

Softmax classifier (SMC) is a supervised model which generalizes logistic regression as

$$
p_{W^{(l)}}\left(y^{l}=i \mid x^{l}\right)=\frac{1}{1+\exp \left(-W^{(l)^{T}} x^{l}\right)},
$$

where $p_{W^{(l)}}(\cdot)(i \in\{0,1\})$ is a sigmoid function with parameters $W^{(l)}$. The input $x^{l}$ of SMC is a high-level feature learned by the DCNN. The parameter $W^{(l)}$ corresponding to the SMC learned via a training set. With the learned parameter $W^{(l)}$, each patch that is fed to the function in Equation (1) produces a value between 0 and 1 that can be interpreted as the probability of 
the input patch corresponding to either EP or ST. The predicted class $\hat{i}$ and prediction score $\hat{s}(\hat{i})$ can then be represented as,

$$
\hat{i}=\left\{i \mid \max _{i} p_{W^{(l)}}\left(y^{l}=i\right)\right\}, \quad i \in\{0,1\}
$$

and

$$
\hat{s}(\hat{i})=p_{W^{(l)}}\left(y^{l}=\hat{i}\right), \quad \hat{i} \in\{0,1\},
$$

respectively.

\subsection{Generating training and testing samples}

Table 2 shows the number of training and testing images for the two data sets $\left(D_{1}\right.$ and $\left.D_{2}\right)$ evaluated in this work. Additional details regarding to $D_{1}$ and $D_{2}$ are provided in Section 4.1.

For the segmentation of the epithelial and stromal compartments for the images in $D_{1}$, the sub-images are generated with two superpixel approaches. The images corresponding to the training and testing sets within $D_{1}$ and $D_{2}$ are initially over-segmented into smaller tissue partitions by superpixel based algorithms [1, 23]. Each tissue compartment is subsequently resized into $50 \times 50$ square sub-image patches via a bicubic interpolation approach [16]. The procedure for generating sub-images for the images from $D_{1}$ is shown in Figs 2 (a)-(d). We use both the Ncut (Normalized cuts) [20, 23] and SLIC (the Simple Linear Iterative Clustering) approach [1]. Our implementations are based on the source code provided in [20, 23]. For comparison, we also employed a fixed-size SW approach for the images in $D_{1}$. Each image in $D_{1}$ is over-segmented into $50 \times 50$ sub-images using a sliding window. The window slides across the entire image row by row from the upper left corner to the lower right with a step size of 25 pixels. Border padding is employed to address issues of boundary artifacts.

For classification of the tissue compartments for the images in $D_{2}$ we employ the approach in [19] to sub-divide the images into $80 \times 80$ square window images using a sliding window. A step size of 40 pixels is employed and as with $D_{1}$. Also border padding is used to avoid boundary artifacts. The procedure for generating sub-images for images from $D_{2}$ is shown in Figures 2 (g)-(i).

The sub-images in the training sets for $D_{1}$ and $D_{2}$ are used for training and optimizing the DCNN based and comparative models (see Table 3 ) while sub-images in the testing sets are used for qualitative and quantitative evaluations. 


\begin{tabular}{|c|c|c|c|c|c|c|c|c|c|c|c|c|c|}
\hline \multirow{4}{*}{\multicolumn{2}{|c|}{ Datasets }} & \multirow{4}{*}{ Total } & \multirow{4}{*}{ Tissue } & \multicolumn{5}{|c|}{ Training } & \multicolumn{5}{|c|}{ Testing } \\
\hline & & & & \multirow{3}{*}{$\begin{array}{l}\sharp \text { of } \\
\text { images }\end{array}$} & \multicolumn{4}{|c|}{$\sharp$ of Sub-images } & \multirow{3}{*}{$\begin{array}{l}\sharp \text { of } \\
\text { images }\end{array}$} & \multicolumn{4}{|c|}{$\sharp$ of Sub-images } \\
\hline & & & & & \multirow{2}{*}{$80 \times 80$} & \multicolumn{3}{|c|}{$50 \times 50$} & & \multirow{2}{*}{$80 \times 80$} & \multicolumn{3}{|c|}{$50 \times 50$} \\
\hline & & & & & & SLIC & Ncut & SW & & & SLIC & Ncut & SW \\
\hline \multirow{4}{*}{$D_{1}$} & \multirow{2}{*}{ NKI } & \multirow{2}{*}{106} & Epithelial & \multirow{2}{*}{69} & & 6763 & 6487 & 5296 & \multirow{2}{*}{37} & & 3657 & 3386 & 2824 \\
\hline & & & Stromal & & & 6935 & 6461 & 5004 & & & 3607 & 3165 & 2659 \\
\hline & \multirow{2}{*}{ VGH } & \multirow{2}{*}{51} & Epithelial & 36 & & 4365 & 3967 & 3585 & \multirow{2}{*}{15} & & 1612 & 1426 & 1475 \\
\hline & & & Stromal & 80 & & 3606 & 3041 & 2779 & & & 1914 & 1340 & 1975 \\
\hline \multirow{2}{*}{\multicolumn{2}{|c|}{$D_{2}$}} & \multirow{2}{*}{1376} & Epithelial & 401 & 15865 & & & & 425 & 27820 & & & \\
\hline & & & Stromal & 255 & 7169 & & & & 295 & 10951 & & & \\
\hline
\end{tabular}

Table 2: The number of training and testing images as well as the corresponding training and testing patches for two superpixel methods (Ncut and SLIC) and sliding window approach for $D_{1}$ and $D_{2}$.

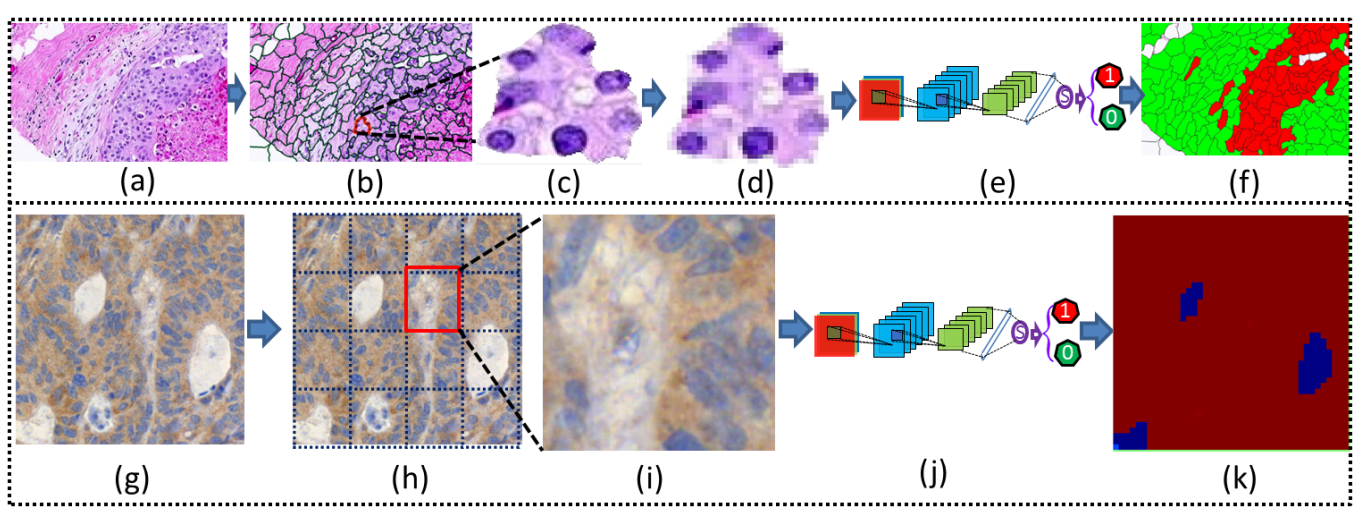

Figure 2: The illustration of DCNN+SMC approach for Epithelial and Stromal segmentation and classification for H\&E (a-f) and IHC (g-k) stained histologic images. The original $\mathrm{H} \& \mathrm{E}$ (a) and $\mathrm{IHC}(\mathrm{g})$ stained images are over-segmented into sub-images using a SLIC (b) and fixed-size square window based approach (h), respectively. An exemplar patch (c) is resized into smaller $50 \times 50$ sub-images $(d)$. The sub-images (d and i) are then fed to a DCNN (e and j) for segmentation and classification of epithelial and stromal regions, shown in panels (f) and (k), respectively.

\section{Experimental Setup}

In order to show the effectiveness of the approach, the DCNN and comparative models are qualitatively and quantitatively evaluated on $D_{1}$ and $D_{2}$, respectively. 


\subsection{Data set}

4.1.1. Data set $1\left(D_{1}\right)$

This data set was downloaded via the links provided in [4]. The data was acquired from two independent cohorts: Netherlands Cancer Institute (NKI) and Vancouver General Hospital (VGH). It consists of 157 rectangular image regions (106 NKI, 51 VGH) in which Epithelial and Stromal regions were manually annotated by pathologists. The images are H\&E stained histologic images from breast cancer TMAs. The size of each image is $1128 \times 720$ pixels at a $20 X$ optical magnification.

\subsubsection{Data set 2 $\left(D_{2}\right)$}

This data was downloaded from the links provided in [19]. The data was originally acquired at the Helsinki University Central Hospital from 1989 to 1998. $D_{2}$ comprises 27 TMAs of colorectal cancer that were stained with epidermal growth factor receptor (EGFR) antibody and hematoxylin counterstain. The slides were digitized with a whole slide scanner under $20 X$ magnification. For the study, a total 1377 rectangular tissue samples of (826 EP and $451 \mathrm{ST}$ ) were chosen from 643 tumor cores. The tissue samples had been previously manually labeled as EP or ST by expert pathologists. The size of the annotations varied between 93-2372 pixels in width and and 942373 in pixel height. As Table 2 shows, the image patches in both $D_{1}$ and $D_{2}$ were approximately evenly divided into training and testing subsets.

\subsection{Training the $D C N N$}

We used a coarse-to-fine sweep approach [27] to choose hyper-parameters for the DCNN. Our approach begins with a coarse setting (wide hyperparameter ranges, training only for 1-5 epochs), to more fine tuned settings (narrow ranges, training with many more epochs). The training procedure is based on the CAFFE framework [15].

\subsection{Parameter setting}

The flowchart illustrated in Figure 2 is applied to both the comparative and DCNN-based approaches for tissue segmentation and classification. Note however that the different approaches differ in terms of the mechanism for feature extraction.

The parameter set for DCNN shown in Figure 1 are as follows. $d_{h}^{1}=20$, $d_{h}^{3}=50, m^{1}=m^{3}=5, \omega^{0}=32, \omega^{1}=28, \omega^{2}=14, \omega^{3}=10, \omega^{4}=5$. The max-pooling operation in the 2 -nd and 4 -th $\mathcal{P}$-layers involves searching over 
each $2 \times 2(s=2)$ neighborhood (see Figure 1(c)). In L5, 50 feature maps of size $5 \times 5$ are fully connected with $d_{h}^{5}=500$ neurons. These 500 neurons are subsequently connected with $d_{h}^{6}=100$ neurons in L6. For the output layer, 100 neurons are fully connected with outputs.

We employ a greedy layer-wise approach [5] for training the DCNN by training each layer in a sequential manner. The trained SMC classifier yields an output based on Equation (1). Based on Equation (2), the class predicted for each input patch is either EP $(\hat{i}=1)$ or ST $(\hat{i}=0)$.

\subsection{Implementation of DCNN and SVM}

All experiments were carried out on a PC (Intel Core(TM) $3.4 \mathrm{GHz}$ processor with 16 GB of RAM) and a Quadro 2000 NVIDIA Graphics Processor Unit. The software implementation was performed using MATLAB 2014a. During the implementation, as Figure 1 shows, we used two $\mathcal{C}$-Layers, $2 \mathcal{P}$ Layers, two full connection layer $(\mathcal{F C}$-Layers), and an output layer. For the $\mathcal{C}$ - and $\mathcal{P}$-Layers, a fixed $5 \times 5$ convolutional and $2 \times 2$ pooling kernel were used.

For the SVM classifier, we employed LIBSVM [7]. The Gaussian kernel was used and 10-fold cross-validation was employed for determining the parameters of the Gaussian kernel.

\subsection{Experimental design}

In the upper block in Figure 2(b), the region with the red contour represents an atomic region generated by the SP algorithm. As DCNN requires uniformly sized sub-images as input, the atomic regions are then resized into $50 \times 50$ square image patches and subsequently fed to the DCNN for model training, segmentation, and classification. Applying this procedure to all atomic regions, the entire input image is classified as being either EP or ST via the DCNN+SMC classifier. For $D_{2}$, the final classification of IHC patches is determined via the averaging of the confidence scores of all the sub-images in each patch.

For the SW based approach, each image pixel is usually segmented twice, the final segmentation result being determined as the most likely of the two segmentation results. To avoid bias in the evaluation process, we used the same image size $(80 \times 80)$ as described in [19] to extract sub-images in $D_{2}$.

\subsection{Comparative strategies}

The DCNN based models and comparative strategies are shown in Table 3. A detailed description of the different models is illustrated in Table 3. 


\begin{tabular}{|c|c|c|c|c|c|c|}
\hline \multirow{2}{*}{ Models } & \multirow{2}{*}{ Feature extraction } & \multicolumn{2}{|c|}{$\begin{array}{l}\text { Generating } \\
\text { sub-images }\end{array}$} & \multicolumn{2}{|c|}{$\begin{array}{c}\text { Size of } \\
\text { Sub-images }\end{array}$} & \multirow{2}{*}{ Classifier } \\
\hline & & $D_{1}$ & $D_{2}$ & $D_{1}$ & $D_{2}$ & \\
\hline Linder[19] & LBP+contrast measure & \multirow{5}{*}{\multicolumn{2}{|c|}{$\begin{array}{l}\text { sliding window } \\
\text { +square image }\end{array}$}} & $50 \times 50$ & \multirow{9}{*}{$80 \times 80$} & \multirow{4}{*}{ SVM } \\
\hline Bianconi $[6]$ & perception-based features & & & & & \\
\hline Color-SW-SVM & pixel intensity & & & \multirow{7}{*}{$50 \times 50$} & & \\
\hline DCNN-SW-SVM & \multirow{6}{*}{ DCNN } & & & & & \\
\hline DCNN-SW-SMC & & & & & & SMC \\
\hline DCNN-Ncut+SMC & & \multirow{4}{*}{$\begin{array}{c}\mathrm{SP}(\mathrm{Ncut})+ \\
\text { square image } \\
\mathrm{SP}(\mathrm{SLIC})+ \\
\text { square image }\end{array}$} & & & & SMC \\
\hline DCNN-Ncut+SVM & & & & & & \multirow{2}{*}{ SVM } \\
\hline DCNN-SLIC+SVM & & & & & & \\
\hline DCNN-SLIC+SMC & & & & & & SMC \\
\hline
\end{tabular}

Table 3: The illustration of models considered in the paper for comparison and the detailed description of the different models.

\subsubsection{DCNN based models versus handcrafted features}

We compared DCNN based approaches with extant handcrafted features used in $[19,6]$. For the color feature based approach (SW-color-SVM), we used color features which includes the color representation of pixel intensities in different color spaces. Linder [19] and Bianconi [6] described two state of art texture feature based approaches to classify EP and ST patches on $D_{2}$, both of which are compared against our DCNN based model.

For $D_{1}$, we implemented the LBP+Contrast measure [19]. Since the authors in [19] did not provide the source code, our implementation is based on the source code provided in [21], which appears to be the approach that inspired the work in [19]. We further compared DCNN+SMC against the approaches in [6] and [19] on $D_{2}$. In order to compare the results of our version of the implementation in [21] the classification results reported in [6] and [19], we linearly mapped the results obtained by the Softmax classifier for the approach we implemented based off $[21]$ from $[0,1]$ to $[-3,3]$. Based on Eqs. (2) and (3), the prediction confidence score is computed by

$$
S\left(\hat{i} \mid x^{l}\right)= \begin{cases}3 \times p_{W^{(l)}}\left(y^{l}=\hat{i} \mid x^{l}\right), & \text { if } \hat{i}=1 \\ -3 \times p_{W^{(l)}}\left(y^{l}=\hat{i} \mid x^{l}\right), & \text { if } \hat{i}=0 .\end{cases}
$$

, where $p_{W^{(l)}}(\cdot)$ is defined in $(1)$. The confidence scores in Figs. 4 and 6 are the computed based on Eq. (4).

\subsubsection{Evaluating DCNN across different sub-image generation methods}

We compared the SP algorithm with fixed-size SW scheme for generating the sub-images. Also, in order to show the performance of different SP 
algorithms in terms of generation of the sub-images, we applied two popular algorithms: Ncut [23] and SLIC [1] for generating the atomic regions.

\subsubsection{Evaluating DCNN with different supervised classifiers}

In order to show the efficiency of DCNN for learning high-level feature, we compared the performance of DCNN coupling to different machine learning classifiers. We compared the DCNN+SMC with the DCNN+SVM. For the DCNN+SVM model, we first trained the DCNN+SMC model with training data. The model parameters for the DCNN model were thus learnt and then fixed for the testing phase. The output of the DCNN was then fed to a Support Vector Machine (SVM) classifier which was then further trained with sub-images in the training set.

\subsubsection{Evaluating the effect of window size on the DCNN}

The segmentation accuracy of the $\mathrm{DCNN}+\mathrm{SMC}$ model was determined in terms of ROC curves, generated across all the test images in $D_{1}$ as a function of the window size.

The performance of the automated segmentation and classification approaches provided by different models is quantified in terms of measurements [22] shown in Table 1. The Receiver Operating Characteristic (ROC) curves are also drawn to assess the classification performance provided by the different models.

\section{Experimental Results and Discussion}

\subsection{Qualitative results}

The qualitative segmentation results of the DCNN (Figure $3(\mathrm{c}-\mathrm{h})$ ) and color feature extraction based (Figure 3(i)) models for a histological image in $D_{1}$ (Figure 3(a)) are shown in Figure 3. In Figures $3(\mathrm{~b}-\mathrm{i})$, green and red regions represent epithelial and stromal regions that were accurately segmented with respect to the pathologist determined ground truth (Figure $3(\mathrm{~b})$ ). The black areas in Figures 3 (b-i) were identified as background regions and hence not worth computationally interrogating. As the qualitative results in Figure $3(\mathrm{c}-\mathrm{h})$ suggest, the segmentation results from the SP-based methods are visually different from the SW-based methods which were prone to producing zigzag boundaries while the SP-based methods produced natural boundaries. Although SP-based methods produced erroneous boundaries as well, the errors appeared more subtle and less egregious, possibly since the superpixel 


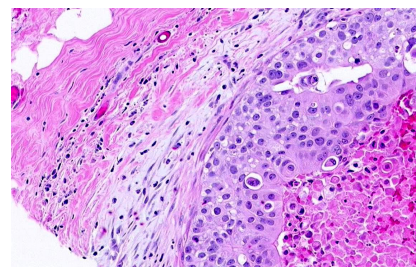

(a)

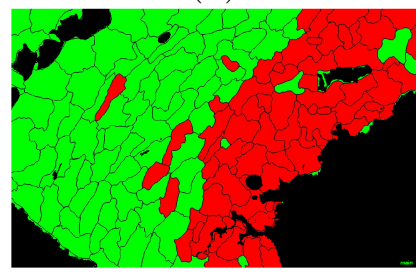

(d)

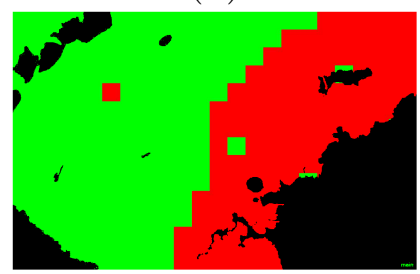

(g)

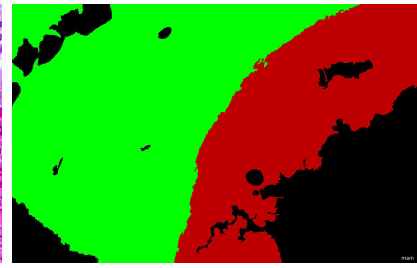

(b)

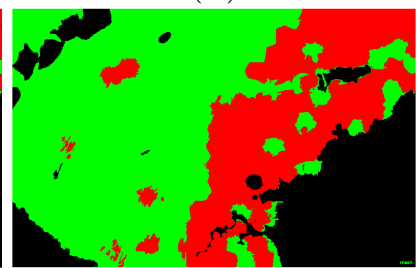

(e)

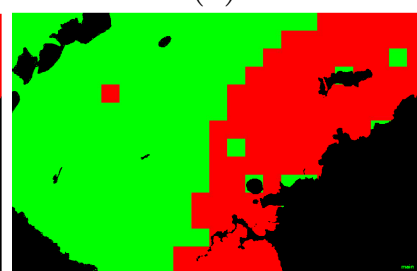

(h)

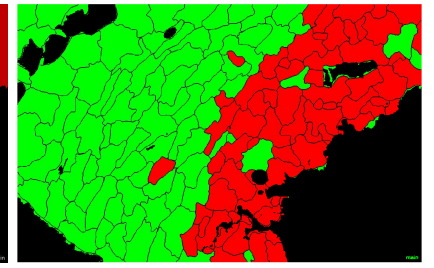

(c)

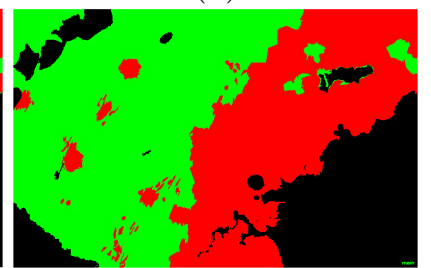

(f)

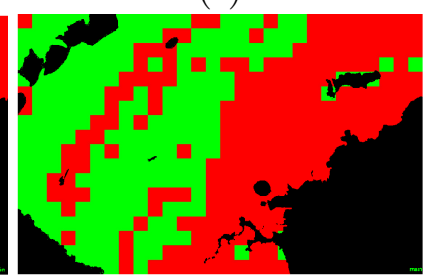

(i)

Figure 3: Segmentation of epithelial (red) and stromal (green) regions on a tissue image (a) using the different segmentation approaches on $D_{1}$. (b) The ground truth (a) annotations of stromal and epithelial regions by an expert pathologist. The classification results are shown for DCNN-Ncut-SVM (c), DCNN-Ncut-SMC (d), DCNN-SLIC-SVM (e), DCNN-SLIC-SMC (f), DCNN-SW-SVM (g), DCNN-SW-SMC (h), and Color-SW-SVM (i), respectively.

based algorithms represent a natural partitioning of visual scenes. The results of pixel-wise classification on EP (Figures $4(\mathrm{a}-\mathrm{e})$ ) and ST (Figures 4 $(\mathrm{f}-\mathrm{k}))$ patches in $D_{2}$ are shown in Figure 4. In Figures $4(\mathrm{a}-\mathrm{k})$, the colors in the heat map (i) correspond to the predicted confidence scores (red $=\mathrm{EP}$ with $100 \%$ and blue $=$ ST with 100\%). The results in Figure 3 appear to suggest that DCNN based models outperform handcrafted feature extraction based models. Also, DCNN+SMC appears to outperform DCNN+SVM. 


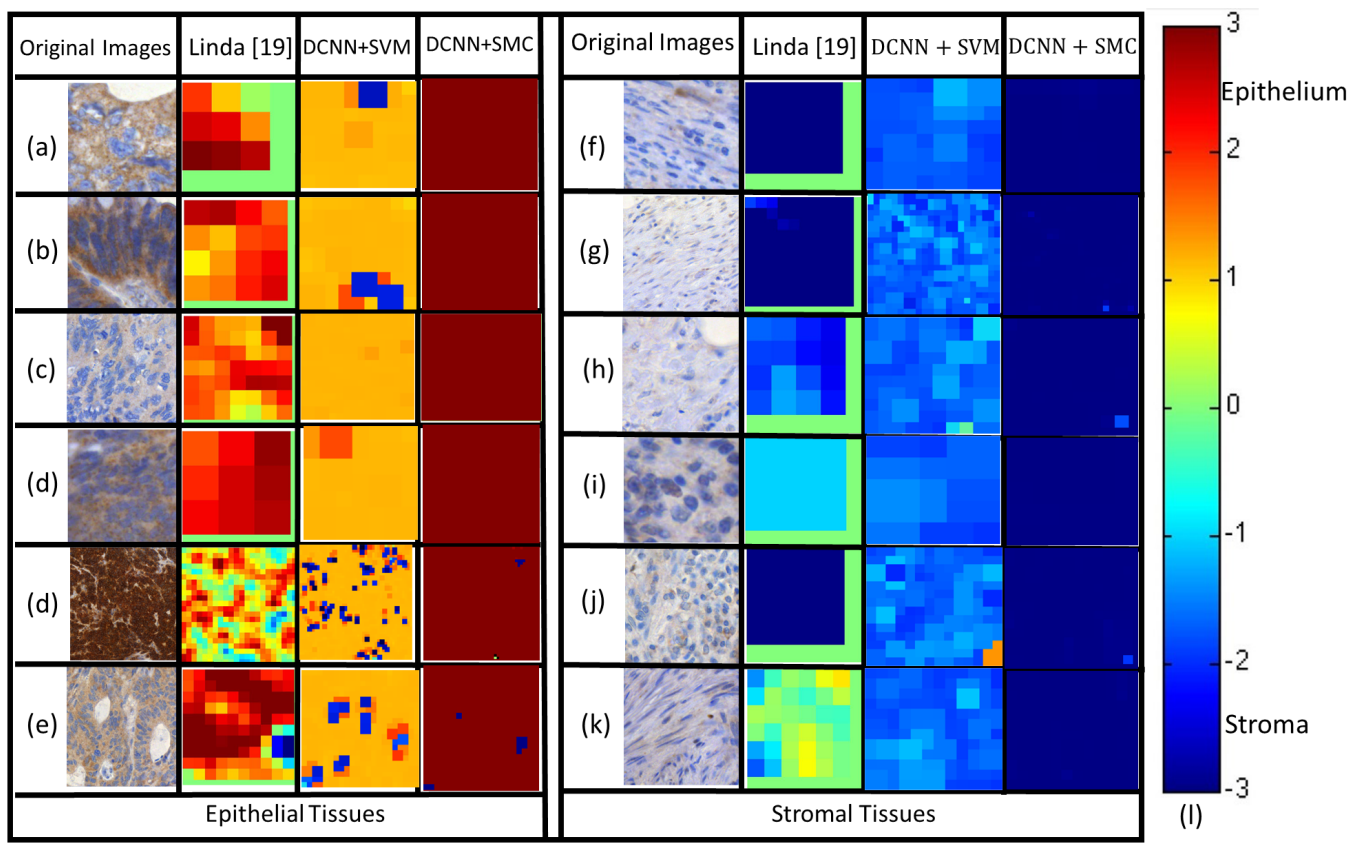

Figure 4: The probability maps rendered by the different DCNN based approaches (Columns 3 and 4) and [19] (in Column 2) for classifying EP ((a)-(e) in the left block, Column 1) and ST ((f)-(k) in the right block, Column 1) patches on $D_{2}$. The false-colour (defined by the heat map (l)) of sub-images in Columns 2-4 reflect the confidence score in predicting them as EP/ST regions via Linda [19], DCNN+SVM, and DCNN+SMC, respectively. The various colors in the heat map (l) correspond to the predicted confidence scores (red = EP with 100\% likelihood and blue $=$ ST with 100\% likelihood).

\subsection{Quantitative results}

The quantitative performance for tissue segmentation and classification for the different models on $D_{1}$ and $D_{2}$ are shown in Table 4 . The DCNN based approach yields a perfect result $(100 \%)$ in terms of True Positive Rate (TPR), True Negative Rate (TNR), Positive Predictive Value (PPV), Negative Predictive Value (NPV), Accuracy (ACC), F1 Score (F1), and Matthews Correlation Coefficient (MCC) and outperforms the approaches described in [6] and [19] respectively. Figures 5 (a) and (b) show the ROC curves corresponding to segmentation accuracy for DCNN-Ncut-SMC, DCNN-SLICSMC, DCNN-Ncut-SVM, DCNN-SLIC-SVM, DCNN-SW-SVM, Color-SWSVM, Linda[19] on NKI (Figure $5(\mathrm{a})$ ) and VGH ((Figure $5(\mathrm{~b})$ ) of $D_{1}$. The 


\begin{tabular}{|c|c|c|c|c|c|c|c|c|c|c|c|c|}
\hline Models & \multicolumn{2}{|c|}{ Datasets } & $\begin{array}{c}\text { TPR } \\
(\%)\end{array}$ & $\begin{array}{c}\text { TNR } \\
(\%)\end{array}$ & $\begin{array}{c}\text { PPV } \\
(\%)\end{array}$ & $\begin{array}{c}\text { NPV } \\
(\%)\end{array}$ & $\begin{array}{c}\text { FPR } \\
(\%)\end{array}$ & $\begin{array}{c}\text { FDR } \\
(\%)\end{array}$ & $\begin{array}{c}\text { FNR } \\
(\%)\end{array}$ & $\begin{array}{c}\mathrm{ACC} \\
(\%)\end{array}$ & $\begin{array}{c}\text { F1 } \\
(\%)\end{array}$ & $\begin{array}{c}\text { MCC } \\
(\%)\end{array}$ \\
\hline \multirow{3}{*}{ Linda[19] } & \multirow[b]{2}{*}{$D_{1}$} & NKI & 46.60 & 90.14 & 83.82 & 60.64 & 9.86 & 16.18 & 53.40 & 67.39 & 59.90 & 40.41 \\
\hline & & VGH & 97.29 & 41.64 & 66.18 & 92.92 & 58.36 & 33.82 & 2.71 & 71.69 & 78.77 & 47.96 \\
\hline & \multicolumn{2}{|c|}{$D_{2}$} & 98.64 & 95.53 & 93.87 & 99.02 & 4.47 & 6.13 & 1.36 & 96.84 & 96.20 & 93.53 \\
\hline \multirow{3}{*}{ Bianconi $[6]$} & \multirow[b]{2}{*}{$D_{1}$} & NKI & & & & & & & & & & \\
\hline & & VGH & & & & & & & & & & \\
\hline & \multicolumn{2}{|c|}{$D_{2}$} & 95.25 & 98.12 & 97.23 & 96.75 & 1.88 & 2.77 & 4.75 & 96.94 & 96.23 & 93.68 \\
\hline \multirow{3}{*}{ DCNN-SW-SVM } & \multirow[b]{2}{*}{$D_{1}$} & NKI & 70.40 & 93.87 & 92.63 & 74.33 & 6.13 & 7.37 & 29.60 & 81.60 & 80.00 & 65.60 \\
\hline & & VGH & 87.01 & 82.20 & 85.15 & 84.36 & 17.80 & 14.85 & 12.99 & 84.79 & 86.07 & 69.36 \\
\hline & \multicolumn{2}{|c|}{$D_{2}$} & 100 & 100 & 100 & 100 & $\mathbf{0}$ & $\mathbf{0}$ & $\mathbf{0}$ & 100 & 100 & 100 \\
\hline \multirow{3}{*}{ Color-SW-SVM } & \multirow[b]{2}{*}{$D_{1}$} & NKI & 89.86 & 58.59 & 70.40 & 84.05 & 41.41 & 29.60 & 10.14 & 74.94 & 78.95 & 51.36 \\
\hline & & VGH & 86.69 & 66.42 & 75.19 & 80.95 & 33.59 & 24.81 & 13.31 & 77.36 & 80.53 & 54.60 \\
\hline & \multicolumn{2}{|c|}{$D_{2}$} & & & & & & & & & & \\
\hline \multirow{3}{*}{ DCNN-SW-SMC } & \multirow[b]{2}{*}{$D_{1}$} & NKI & 77.95 & 80.68 & 81.63 & 76.86 & 19.32 & 18.37 & 22.05 & 79.25 & 79.75 & 58.56 \\
\hline & & VGH & 82.18 & 86.12 & 87.46 & 80.40 & 13.88 & 12.54 & 17.82 & 83.99 & 84.74 & 68.08 \\
\hline & \multicolumn{2}{|c|}{$D_{2}$} & & & & & & & & & & \\
\hline \multirow{3}{*}{ DCNN-Ncut-SVM } & \multirow[b]{2}{*}{$D_{1}$} & NKI & 81.09 & 86.39 & 86.71 & 80.67 & 13.61 & 13.27 & 18.91 & 83.62 & 83.81 & 67.43 \\
\hline & & VGH & 88.29 & 88.40 & 89.93 & 86.55 & 11.60 & 10.07 & 11.71 & 88.34 & 89.10 & 76.59 \\
\hline & \multicolumn{2}{|c|}{$D_{2}$} & & & & & & & & & & \\
\hline \multirow{3}{*}{ DCNN-Ncut-SMC } & \multirow[b]{2}{*}{$D_{1}$} & NKI & 88.92 & 67.94 & 75.23 & 84.85 & 32.06 & 24.77 & 11.08 & 78.91 & 81.05 & 58.45 \\
\hline & & VGH & 89.37 & 86.63 & 88.67 & 87.42 & 13.37 & 11.31 & 10.63 & 88.11 & 89.03 & 76.05 \\
\hline & \multicolumn{2}{|c|}{$D_{2}$} & & & & & & & & & & \\
\hline \multirow{3}{*}{ DCNN-SLIC-SVM } & \multirow[b]{2}{*}{$D_{1}$} & NKI & 80.63 & 85.79 & 86.13 & 80.18 & 14.21 & 13.87 & 19.37 & 83.09 & 83.29 & 66.37 \\
\hline & & VGH & 88.51 & 83.68 & 86.41 & 86.13 & 16.32 & 13.59 & 11.49 & 86.28 & 87.45 & 72.36 \\
\hline & \multicolumn{2}{|c|}{$D_{2}$} & & & & & & & & & & \\
\hline \multirow{3}{*}{ DCNN-SLIC-SMC } & \multirow{3}{*}{$D_{1}$} & NKI & 86.31 & 82.15 & 84.11 & 84.60 & 17.85 & 15.89 & 13.66 & 84.34 & 85.21 & 68.60 \\
\hline & & VGH & 87.88 & 82.13 & 85.22 & 85.25 & 17.87 & 14.78 & 12.12 & 85.23 & 86.53 & 70.24 \\
\hline & & $D_{2}$ & & & & & & & & & & \\
\hline
\end{tabular}

Table 4: The quantitative evaluation of segmentation and classification results on $D_{1}$ and $D_{2}$ with different models. The bolded numbers reflect the best performance for a specific performance measure within the data set.

AUC values suggest that the DCNN based models outperform the handcrafted feature based approaches (Color-SW-SVM model and approaches in [19]), with the DCNN-Ncut-SVM is emerging as marginally better than the other models. Figure 6 shows the histogram for the DCNN-SW-SVM model for $\mathrm{EP}$ and ST patch based classification for $D_{2}$. Figure 6 is a plot of number of images (Y-axis) versus the confidence score (X-axis) for the SVM classifier. The two types of image patches appear to be well separated. Finally, in terms of the comparison between two different SP algorithms, Table 4 shows that the performance of Ncut is slightly better than SLIC on $D_{1}$. Additionally, the SVM classifier slightly outperforms SMC on $D_{1}$.

\subsection{Sensitivity Analysis}

Figure 7 shows the sensitivity of window size (X-axis) on the segmentation accuracy (Y-axis) for the DCNN-SW-SVM model $D_{1}$. Figure 7 suggests that 


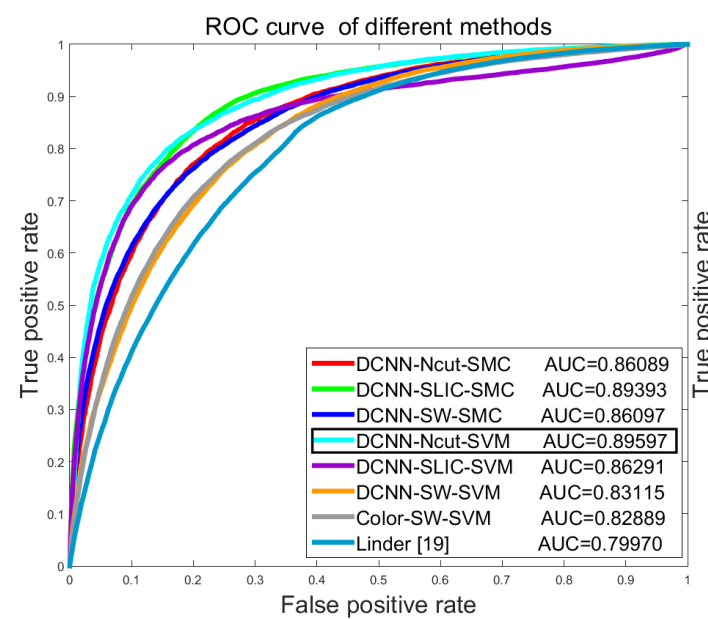

(a)

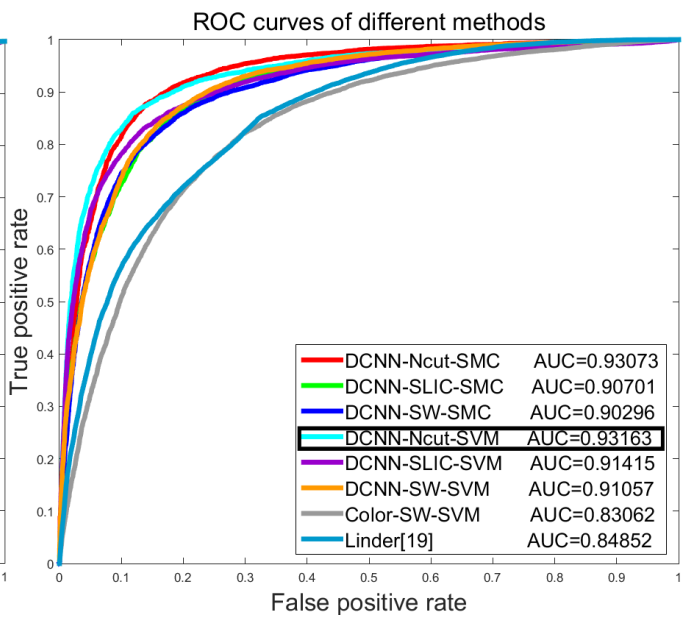

(b)

Figure 5: The ROC curves for the different models (see Table 3) for detecting EP and ST regions on NKI (a) and VGH (b) data cohorts within $D_{1}$.

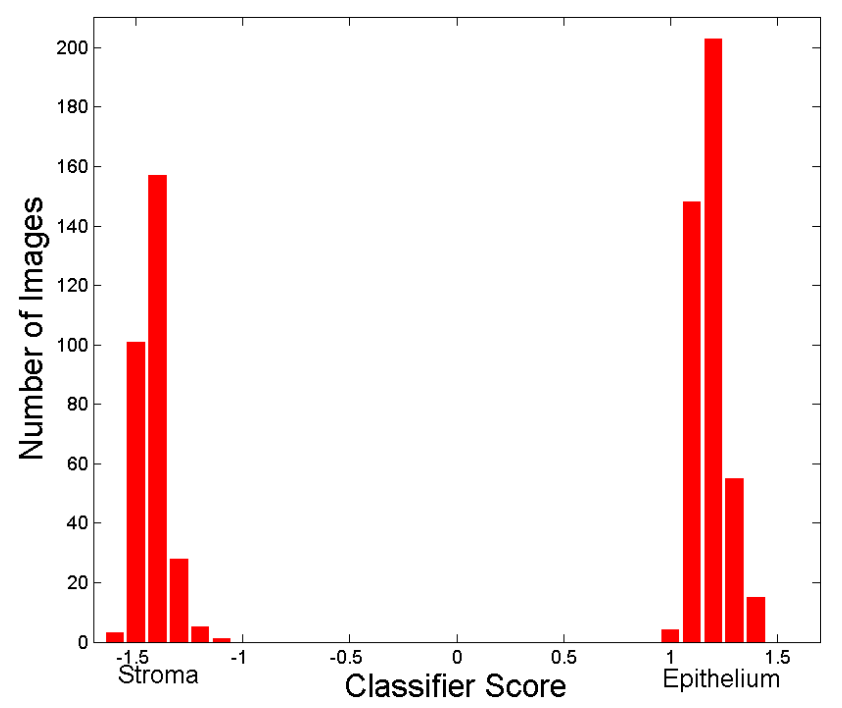

Figure 6: A plot of the score value on the $\mathrm{X}$-axis versus the number of image patches on the Y-axis for the model DCNN-SW-SVM on dataset $D_{2}$.

the DCNN-SW-SVM model achieves the best AUC value when the window size is around $50 \times 50$ pixels. As a result, we chose a window size of $50 \times 50$ 
for all our subsequent experiments.

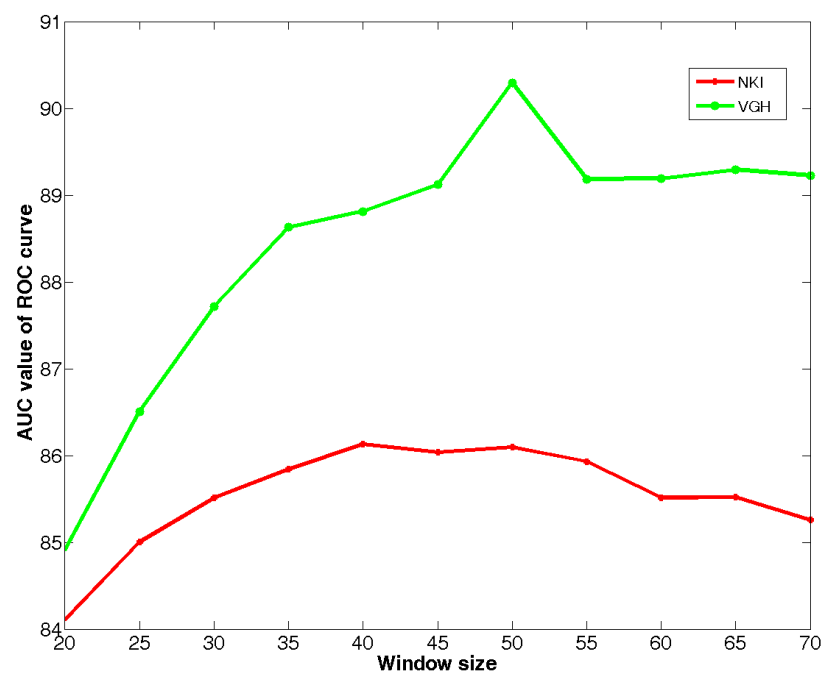

Figure 7: Plot of AUC versus window size for the DCNN-SW-SVM model on NKI and VGH datasets within $D_{1}$.

\section{Concluding Remarks}

In this paper we presented a new Deep Convolutional Neural Network (DCNN) based model for segmentation and classification of epithelial and stromal regions within Hematoxylin and Eosin ( $\mathrm{H} \& \mathrm{E}$ ) and Immunohistochemistry (IHC) images of breast and colon cancer. DCNN uses a deep architecture to learn complex features in a data-driven fashion and that has been shown in multiple applications outperform the classification accuracy obtained via handcrafted features. We compared the DCNN based models with extant handcrafted features and showed that for the task of separating stroma from epithelium, the DCNN based models consistently outperformed handcrafted features based models. Future work will entail evaluation of our approach on tissue partitioning for other types of cancers as well.

\section{Acknowledgement}

Research reported in this publication was supported by National Natural Science Foundation of China (No. 61273259); Six Major Talents Summit 
of Jiangsu Province (No. 2013-XXRJ-019), and Natural Science Foundation of Jiangsu Province of China (No. BK20141482); Jiangsu Innovation \& Entrepreneurship Group Talents Plan; the National Cancer Institute of the National Institutes of Health under award numbers R01CA136535-01, R01CA140772-01, R21CA167811-01, R21CA179327-01; the National Institute of Diabetes and Digestive and Kidney Diseases under award number R01DK098503-02, the DOD Prostate Cancer Synergistic Idea Development Award (PC120857); the DOD Lung Cancer Idea Development New Investigator Award (LC130463), the Ohio Third Frontier Technology development Grant, the CTSC Coulter Annual Pilot Grant, and the Wallace H. Coulter Foundation Program in the Department of Biomedical Engineering at Case Western Reserve University.

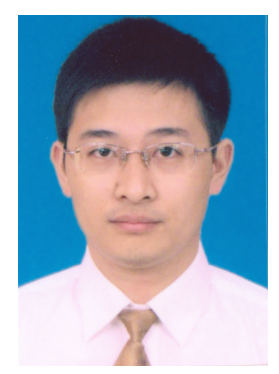

Dr. Jun Xu received his M.S. degree in applied mathematics from the University of Electronic Science and Technology of China, Chengdu, China, in 2004, and the Ph.D. degree in control science and engineering from Zhejiang University, Hangzhou, China, in 2007. From 2008 to 2011, he had been a postdoctoral associate and research assistant at Dept. of Biomedical Engineering, Rutgers University, USA. In the summer of 2014, he had been a Visiting Assistant Professor at Dept. of Biomedical Engineering, Case Western Reserve University, USA. He is currently a full professor at School of Information \& Control, Nanjing University of Information Science and Technology, China. His research interests include medical image analysis, computer vision, machine learning, digital pathology, and computer-aided detection, diagnosis, and prognosis on cancer. He authored more than thirty papers in various leading international journals and conferences. His research work has received grant funding from National Science Foundation of China.

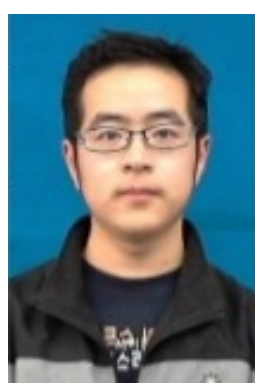

Mr. Xiaofei Luo received the B.S. degrees from the Nanjing University of Information Science and Technology, Nanjing, China, in 2013. He is currently working toward the M.S. degree in the School of Information and Control, Nanjing University of Information Science and Technology. His current research interests is machine learning and its application in computer-aided diagnosis on cancers. 


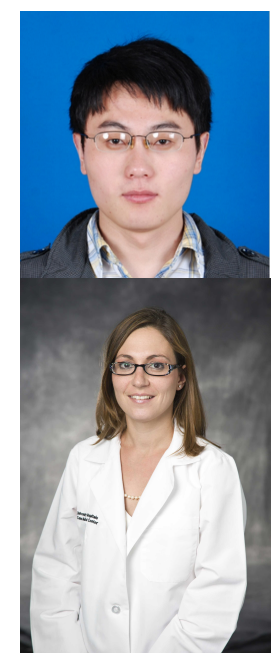

Mr. Guanhao Wang received his B.S. and M.S. degrees from the Nanjing University of Information Science and Technology, Nanjing, China, in 2012 and 2015, respectively. His research interest is pattern recognition. He is now working at Novatek Inc. in Shanghai, China.

Dr. Hannah Gilmore MD is the Director of Surgical Pathology and Director of the Breast Pathology Service at University Hospitals Case Medical Center. She is an Assistant Professor in the Department of Pathology at Case Western Reserve University and is a member of the Case Comprehensive Cancer Center. She is a clinical expert in the diagnosis of breast diseases and is a member of the College of American Pathologists Advanced Multidisciplinary Breast Program. Dr. Gilmores research in breast disease spans the clinical, translational and basic science spectrum and has been published in numerous peer-reviewed journals. In addition to receiving research and teaching awards, her work has been funded by the National Cancer Institute, the Department of Defense, the American Cancer Society as well as from Industry.

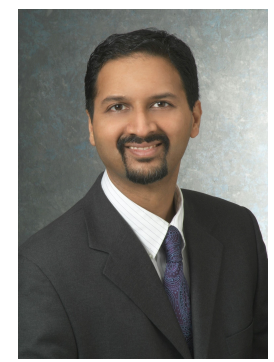

Dr. Anant Madabhushi is the Director of the Center for Computational Imaging and Personalized Diagnostics (CCIPD) and a Professor in the Departments of Biomedical Engineering, Pathology, Radiology, Radiation Oncology, Urology, General Medical Sciences, and Electrical Engineering and Computer Science at Case Western Reserve University. Dr. Madabhushi has authored over 240 peer-reviewed publications in leading international journals and conferences. He has 10 issued patents with over 20 patents pending in the areas of medical image analysis, computer-aided diagnosis, and computer vision. He is an Associate Editor for IEEE Transactions on Biomedical Engineering, IEEE Transactions on Biomedical Engineering Letters, BMC Cancer, BMC Medical Imaging, Journal of Medical Imaging and Medical Image Analysis (MedIA). He is also on the Editorial Board of the Journal Analytical and Cellular Pathology. He has been the recipient of a number of awards for both research as well as teaching, including the Department of Defense New Investigator Award in Lung Cancer (2014), the Coulter Phase 1 and Phase 2 Early Career award (2006, 2008), and the Excellence in Teaching Award (2007-2009), along with a number of technology commercialization awards. He is also a Wallace H. 
Coulter Fellow, a Fellow of the American Institute of Medical and Biological Engineering (AIMBE), and a Senior IEEE member. In 2015 he was named by Crains Cleveland Business Magazine as one of Forty under 40 making positive impact to business in North East Ohio. His research work has received grant funding from the National Cancer Institute (NIH), National Science Foundation, the Department of Defense, private foundations, and from Industry.

He is also the co-founder of Ibris Inc. a startup company focused on developing image based assays for breast cancer prognosis. He is also the conference chair for the new Digital Pathology Conference to be held annually in conjunction with the SPIE Medical Imaging Symposium. 
[1] Achanta, R., Shaji, A., Smith, K., Lucchi, A., Fua, P., Susstrunk, S., 2012. Slic superpixels compared to state-of-the-art superpixel methods. Pattern Analysis and Machine Intelligence, IEEE Transactions on 34 (11), 2274-2282.

[2] Ali, S., et al., 2013. Spatially aware cell clusters graphs: Predicting outcome in hpv associated oropharyngeal tumors. In: Med Image Comput Comput Assist Interv. Vol. 8149. pp. 412-519.

[3] Amaral, T., McKenna, S., Robertson, K., Thompson, A., Oct 2013. Classification and immunohistochemical scoring of breast tissue microarray spots. Biomedical Engineering, IEEE Transactions on 60 (10), 28062814.

[4] Beck, A. H., at el., 2011. Systematic analysis of breast cancer morphology uncovers stromal features associated with survival. Science Translational Medicine 3 (108), 108ra113.

[5] Bengio, Y., Lamblin, P., Popovici, D., Larochelle, H., et al., 2007. Greedy layer-wise training of deep networks. Advances in neural information processing systems 19, 153.

[6] Bianconi, F., lvarez Larrn, A., Fernndez, A., 2015. Discrimination between tumour epithelium and stroma via perception-based features. Neurocomputing 154 (0), 119 - 126.

URL http: //www.sciencedirect.com/science/article/pii/S0925231214016762

[7] Chang, C.-C., Lin, C.-J., 2011. LIBSVM: A library for support vector machines. ACM Transactions on Intelligent Systems and Technology 2, 27:1-27:27, software available at http://www.csie.ntu.edu.tw/ cjlin/libsvm.

[8] Chang, H., Zhou, Y., Borowsky, A., Barner, K., Spellman, P., Parvin, B., 2015. Stacked predictive sparse decomposition for classification of histology sections. International Journal of Computer Vision 113 (1), $3-18$.

URL http://dx.doi.org/10.1007/s11263-014-0790-9

[9] Ciresan, D. C., et al., 2013. Mitosis detection in breast cancer histology images with deep neural networks. In: MICCAI 2013. Vol. 8150 of LNCS. Springer, pp. 411-418. 
[10] Cruz-Roa, A., et al., 2013. A deep learning architecture for image representation, visual interpretability and automated basal-cell carcinoma cancer detection. In: MICCAI 2013. Vol. 8150. Springer, pp. 403-410.

[11] De Wever, O., Mareel, M., 2003. Role of tissue stroma in cancer cell invasion. The Journal of pathology 200 (4), 429-447.

[12] Downey, C. L., Simpkins, S. A., White, J., Holliday, D. L., Jones, J. L., Jordan, L. B., Kulka, J., Pollock, S., Rajan, S. S., Thygesen, H. H., Hanby, A. M., Speirs, V., Apr. 2014. The prognostic significance of tumour-stroma ratio in oestrogen receptor-positive breast cancer. Br J Cancer 110 (7), 1744-1747.

URL http://dx.doi.org/10.1038/bjc.2014.69

[13] Eramian, M., et al., 2011. Segmentation of epithelium in h\&e stained odontogenic cysts. Journal of microscopy 244 (3), 273-292.

[14] Hiary, H., et al., 2013. Automated segmentation of stromal tissue in histology images using a voting bayesian model. Signal, Image and Video Processing 7 (6), 1229-1237.

URL http://dx.doi.org/10.1007/s11760-012-0393-2

[15] Jia, Y., Shelhamer, E., Donahue, J., Karayev, S., Long, J., Girshick, R., Guadarrama, S., Darrell, T., 2014. Caffe: Convolutional architecture for fast feature embedding. arXiv preprint arXiv:1408.5093.

[16] Keys, R., Dec 1981. Cubic convolution interpolation for digital image processing. Acoustics, Speech and Signal Processing, IEEE Transactions on 29 (6), 1153-1160.

[17] Lahrmann, B., Halama, N., Sinn, H.-P., Schirmacher, P., Jaeger, D., Grabe, N., 12 2011. Automatic tumor-stroma separation in fluorescence tmas enables the quantitative high-throughput analysis of multiple cancer biomarkers. PLoS ONE 6 (12), e28048.

[18] LeCun, Y., Bengio, Y., Hinton, G., May 2015. Deep learning. Nature 521 (7553), 436-444.

URL http://dx.doi.org/10.1038/nature14539

[19] Linder, N., et al., 2012. Identification of tumor epithelium and stroma in tissue microarrays using texture analysis. Diagnostic Pathology 7 (1), 
22.

URL http://www.diagnosticpathology.org/content/7/1/22

[20] Madabhushi, A., Shi, J., Rosen, M., Tomaszeweski, J., Feldman, M., 2005. Graph embedding to improve supervised classification and novel class detection: Application to prostate cancer. In: Duncan, J., Gerig, G. (Eds.), Medical Image Computing and Computer-Assisted Intervention ? MICCAI 2005. Vol. 3749 of Lecture Notes in Computer Science. Springer Berlin Heidelberg, pp. 729-737.

[21] Ojala, T., Pietikainen, M., Maenpaa, T., Jul 2002. Multiresolution grayscale and rotation invariant texture classification with local binary patterns. Pattern Analysis and Machine Intelligence, IEEE Transactions on 24 (7), 971-987.

[22] Powers, D., 2007. Evaluation: From precision, recall and f-factor to roc, informedness, markedness \& correlation (tech. rep.). Adelaide, Australia.

[23] Ren, X., Malik, J., 2003. Learning a classification model for segmentation. In: ICCV2003. pp. 10-17 vol.1.

[24] Shi, J., Malik, J., 2000. Normalized cuts and image segmentation. Pattern Analysis and Machine Intelligence, IEEE Transactions on 22 (8), 888-905.

[25] Su, H., Xing, F., Kong, X., Xie, Y., Zhang, S., Yang, L., 2015. Robust cell detection and segmentation in histopathological images using sparse reconstruction and stacked denoising autoencoders. In: Navab, N., Hornegger, J., Wells, W., Frangi, A. (Eds.), Medical Image Computing and Computer-Assisted Intervention: MICCAI 2015. Vol. 9351 of Lecture Notes in Computer Science. Springer International Publishing, pp. 383-390.

[26] Wang, H., et al., 2014. Mitosis detection in breast cancer pathology images by combining handcrafted and convolutional neural network features. Journal of Medical Imaging 1 (3), 034003.

URL http://dx.doi.org/10.1117/1.JMI.1.3.034003 
[27] Weller, D. S., Ramani, S., Nielsen, J.-F., Fessler, J. A., 2014. Monte carlo sure-based parameter selection for parallel magnetic resonance imaging reconstruction. Magnetic Resonance in Medicine 71 (5), 1760-1770.

[28] Xu, J., Xiang, L., Hang, R., Wu, J., 2014. Stacked sparse autoencoder (ssae) based framework for nuclei patch classification on breast cancer histopathology. In: Biomedical Imaging (ISBI), 2014 IEEE 11th International Symposium on. IEEE, pp. 999-1002.

[29] Xu, J., Xiang, L., Liu, Q., Gilmore, H., Wu, J., Tang, J., Madabhushi, A., Jan 2016. Stacked sparse autoencoder (ssae) for nuclei detection on breast cancer histopathology images. Medical Imaging, IEEE Transactions on 35 (1), 119-130.

[30] Xu, J., Xiang, L., Wang, G., Ganesan, S., Feldman, M., Shih, N. N., Gilmore, H., Madabhushi, A., 2015. Sparse non-negative matrix factorization (snmf) based color unmixing for breast histopathological image analysis. Computerized Medical Imaging and Graphics 46, Part 1, 20 - 29, sparsity Techniques in Medical Imaging.

URL http://www. sciencedirect.com/science/article/pii/S0895611115000774

[31] Yuan, Y., et al., 2012. Quantitative image analysis of cellular heterogeneity in breast tumors complements genomic profiling. Science Translational Medicine 4 (157), 157ra143.

[32] Zhang, X., Liu, W., Dundar, M., Badve, S., Zhang, S., Feb 2015. Towards large-scale histopathological image analysis: Hashing-based image retrieval. Medical Imaging, IEEE Transactions on 34 (2), 496-506.

[33] Zhang, X., Liu, W., Zhang, S., April 2014. Mining histopathological images via hashing-based scalable image retrieval. In: Biomedical Imaging (ISBI), 2014 IEEE 11th International Symposium on. pp. 1111-1114.

[34] Zhang, X., Su, H., Yang, L., Zhang, S., June 2015. Fine-grained histopathological image analysis via robust segmentation and large-scale retrieval. In: The IEEE Conference on Computer Vision and Pattern Recognition (CVPR). 